\title{
HOLOMORPHIC SOLUTIONS OF FUNCTIONAL DIFFERENTIAL SYSTEMS NEAR SINGULAR POINTS
}

\author{
L. J. GRIMM ${ }^{1}$ AND L. M. HALL
}

\begin{abstract}
Functional analysis techniques are used to prove a theorem, analogous to the Harris-Sibuya-Weinberg theorem for ordinary differential equations, which yields as corollaries a number of existence theorems for holomorphic solutions of linear functional differential systems of the form $z^{D} y^{\prime}(z)=A(z) y(z)+$ $B(z) y(\alpha z)+C(z) y^{\prime}(\alpha z)$ in the neighborhood of the singularity at $z=0$.
\end{abstract}

The existence of holomorphic solutions of ordinary differential systems near a singular point has been extensively studied. F. Lettenmeyer [6] showed that a linear system with an irregular singular point at $z=z_{0}$ may have several linearly independent solutions holomorphic at $z_{0}$; his theorem gives an estimate on the number of such solutions. Lettenmeyer's original proof was quite involved; the proof has been greatly simplified by W. A. Harris, Jr., Y. Sibuya, and L. Weinberg [5], who used functional analysis techniques to establish a theorem which includes Lettenmeyer's theorem and several results on systems of Briot-Bouquet type as simple corollaries.

Several authors ([1], [2], [3], [7]) have studied existence of solutions of functional differential equations with contracting arguments in the neighborhood of a singularity at the origin. All the equations considered in their articles are of Briot-Bouquet type, and only Grudo in [3] deals with systems of neutral-differential equations. In this paper we extend the results of Harris, Sibuya and Weinberg to a class of neutral-differential systems, obtaining as corollaries an analogue of Lettenmeyer's theorem and a generalization of the results of Grudo. Our principal result is the following theorem.

THEOREM. Let $A(z), B(z)$, and $C(z)$ be $n \times n$ matrices holomorphic at $z=0$, let $D=\operatorname{diag}\left(d_{1}, \cdots, d_{n}\right)$ with nonnegative integers $d_{i}$, and let $\alpha,|\alpha|<1$, be a complex constant. Then for every positive integer $N$ sufficiently large, and every polynomial $\phi(z)$ with $z^{D} \phi(z)$ of degree $N$, there exists a polynomial $f(z ; \phi)$ (depending on $A, B, C, \alpha$, and $N$ ) of degree

Received by the editors March 28, 1973.

AMS (MOS) subject classifications (1970). Primary 34K05; Secondary 30A20, $34 \mathrm{~A} 20$.

${ }^{1}$ Research supported by NSF Grant GP-27628. 
$N-1$ such that the linear neutral-differential system

$$
z^{D} y^{\prime}(z)=A(z) y(z)+B(z) y(\alpha z)+C(z) y^{\prime}(\alpha z)+f(z ; \phi)
$$

has a solution $y(z)$ holomorphic at $z=0$ Further, $f$ and $y$ are linear and homogeneous in $\phi$, and $z^{D}(y-\phi)=O\left(z^{N+1}\right)$ as $z \rightarrow 0$.

Proof. The proof is an application of the Banach fixed point theorem. Let $\delta>0$ and let $X$ be the set of all $n$-vector valued functions $f=f(z)$ whose components have absolutely convergent power series expansions in $|z| \leqq \delta$. For $f \in X, f(z)=\sum_{k=0}^{\infty} f_{k} z^{k}, f_{k}=\left(f_{k}^{1}, \cdots, f_{k}^{n}\right)^{T}$, define $\|f\|=\sum_{k=0}^{\infty}\left|f_{k}\right| \delta^{k}$, where $\left|f_{k}\right|=\sum_{j=1}^{n}\left|f_{k}^{j}\right|$. With this norm, $X$ is a Banach space.

For a sufficiently large positive integer $N$, define the mapping $L_{N}: X \rightarrow X$ as follows: $L_{N} y=g$, where

$$
y(z)=\left(y^{1}(z), \cdots, y^{n}(z)\right)^{T}, \quad g(z)=\left(g^{1}(z), \cdots, g^{n}(z)\right)^{T},
$$

with $y^{j}(z)=\sum_{k=0}^{\infty} y_{k}^{j} z^{k}, g^{j}(z)=\sum_{k=N}^{\infty}\left(y_{k}^{j} z^{k+1-d_{j}}\right) /\left(k+1-d_{j}\right)$. Hence

$$
\left\|L_{N} y\right\| \leqq \sum_{j=1}^{n} \frac{\delta^{1-d j}}{N+1-d_{j}}\|y\| .
$$

Define $\hat{y}(z)=\left(y^{1}(\alpha z), \cdots, y^{n}(\alpha z)\right)^{T} \equiv\left(\hat{y}^{1}(z), \cdots, \hat{y}^{n}(z)\right)^{T}$ with

$$
\hat{y}^{j}(z)=\sum_{k=0}^{\infty} \hat{y}_{k} z^{k} \equiv \sum_{k=0}^{\infty} y_{k}^{j} \alpha^{k} z^{k}
$$

Also define $y^{*}(z)=\left(y^{* 1}(z), \cdots, y^{* n}(z)\right)^{T}$, with

$$
y^{* j}(z)=\sum_{k=0}^{\infty}(k+1) \alpha^{k} y_{k+1}^{j} z^{k}
$$

Note that $\hat{y}$ and $y^{*}$ have absolutely convergent power series expansions for $|z| \leqq \delta$, and also that

$$
\|\hat{y}\| \leqq\|y\| .
$$

Furthermore, setting $\chi(z)=\sum_{k=0}^{\infty}\left(\sum_{j=1}^{n}\left|y_{k}^{j}\right|\right) z^{k},|z| \leqq \delta$, we have

$$
\chi^{\prime}(|\alpha| z)=\sum_{k=1}^{\infty} k\left(\sum_{j=1}^{n}\left|y_{k}^{j}\right|\right)|\alpha|^{k-1} z^{k-1}, \quad|z| \leqq \delta .
$$

By the Cauchy integral formula,

$$
\left|\chi^{\prime}(|\alpha| z)\right| \leqq \frac{\max _{|\zeta|=\delta}|\chi(\zeta)|}{\delta^{2}(1-|\alpha|)^{2}}=\frac{\|y\|}{\delta^{2}(1-|\alpha|)^{2}}, \quad|z| \leqq \delta .
$$


Hence

$$
\left\|y^{*}\right\|=\left|\chi^{\prime}(|\alpha| \delta)\right| \leqq\|y\| / \delta^{2}(1-|\alpha|)^{2} .
$$

If $M$ is an $n \times n$ matrix, $M=\left(m^{i j}\right)$, with elements having absolutely convergent power series expansions for $|z| \leqq \delta, m^{i j}=\sum_{k=0}^{\infty} m_{k}^{i j} z^{k}$, then for $f \in X$ we have $M f \in X$ and $\|M f\| \leqq\|M\|\|f\|$, where

$$
\|M\|=\sum_{i, j=1}^{n}\left(\sum_{k=0}^{\infty}\left|m_{k}^{i j}\right| \delta^{k}\right) \text {. }
$$

Let $\phi=\left(\phi^{1}, \cdots, \phi^{n}\right)^{T}$ be a vector polynomial with $\phi^{j}(z)=\sum_{k=0}^{j-d_{j}} \phi_{k}^{j} z^{k}$, and consider the functional equation in $X$

$$
y=\phi+T_{N}[y]
$$

where $T_{N}[y]=L_{N}\left(A y+B \hat{y}+C y^{*}\right)$. The estimates (2)-(4) imply that for $N$ sufficiently large, $\left\|T_{N}\right\|<1$, and thus there exists a unique solution $y \in X, y(\cdot ; \phi)=\left(I-T_{N}\right)^{-1} \phi$.

From the definition of the mapping $T_{N}$ it follows that the holomorphic solution of the functional equation (5) satisfies the linear differential system of the form (1), where

$$
\begin{aligned}
f(z ; \phi) & \equiv \sum_{k=0}^{N-1} f_{k} z^{k} \\
& \equiv z^{D} \frac{d \phi}{d z}-\sum_{k=0}^{N-1} A y(\cdot ; \phi)_{k} z^{k}-\sum_{k=0}^{N-1} B \hat{y}(\cdot ; \phi)_{k} z^{k}-\sum_{k=0}^{N-1} C y^{*}(\cdot ; \phi)_{k} z_{k} .
\end{aligned}
$$

Since the coefficients of $y(\cdot ; \phi)$ (and thus also $\hat{y}$ and $y^{*}$ ) are linear in the coefficients of $\phi$, this is also true for the $f_{k}$. The proof is complete.

The corollaries below follow from the theorem similarly to the proofs of corresponding results in [5].

Corollary 1. Let $D=\operatorname{trace} D$ and $n-d \geqq 0$. Then the system

$$
z^{D} y^{\prime}(z)=A(z) y(z)+B(z) y(\alpha z)+C(z) y^{\prime}(\alpha z)
$$

has at least $n-d$ linearly independent solutions holomorphic at $z=0$.

COROLlary 2. Let

$$
A(z)=\sum_{k=0}^{\infty} A_{k} z^{k}, \quad B(z)=\sum_{k=0}^{\infty} B_{k} z^{k}, \quad \text { and } \quad C(z)=\sum_{k=1}^{\infty} C_{k} z^{k}
$$

be convergent for $|z|<a(a>0)$, and let $y(z)=\sum_{k=0}^{\infty} y_{k} z^{k}$ be a formal solution of

$$
z y^{\prime}(z)=A(z) y(z)+B(z) y(\alpha z)+C(z) y^{\prime}(\alpha z) .
$$

Then $y(z)$ is convergent for $|z|<a$. 
Corollary 3. Let $A, B$, and $C$ be as in Corollary 2, let $m$ be a fixed integer, let $\alpha \neq 0$, and let $n_{m+k}$ be the number of linearly independent eigenvectors corresponding to the eigenvalue $m+k$ of the matrix

$$
\mathfrak{U}_{m+k}=\left[A_{0}+\alpha^{m+k} B_{0}+(m+k) \alpha^{m+k-1} C_{1}\right] .
$$

Then the number $N_{m}(\geqq 0)$ of linearly independent solutions of the differential system (8) of the form $y=\sum_{k=0}^{\infty} y_{k} z^{m+k}$ satisfies $N_{m} \leqq n_{m}+n_{m+1}+\cdots$. If, in addition, $B_{0}=C_{1}=0$, then $N_{m} \geqq \max \left(n_{m}, n_{m+1}, \cdots\right)$.

REMARKS. 1. The results extend without change to systems with several deviating arguments of the same form.

2. If $A_{0}=B_{0}=C_{0}=0$, then $z=0$ is an ordinary point for the system (8). Hence, by Corollary 1 , there exist at least $n$ linearly independent solutions for this system. If, in addition, $C_{1}=0$, then the coefficients of each formal solution are determined recursively and there exist exactly $n$ linearly independent solutions of the system holomorphic at $z=0$.

3. Analogous results hold for nonlinear systems of the form

$$
z^{D} y^{\prime}(z)=h\left(z, y(z), y(\alpha z), y^{\prime}(\alpha z)\right)+f(z ; \phi)
$$

and can be obtained by considerations similar to those in the paper of Harris [4].

\section{REFERENCES}

1. L. E. El'sgol'c, Equations with retarded argument similar to Euler's equation, Trudy Sem. Teor. Differencial. Uravenii s Otklon. Argumentom Univ. Družby Narodov Patrisa Lumumby 1 (1962), 120. (Russian) MR 32 \#2699.

2. L. J. Grimm, Analytic solutions of a neutral differential system near a singular point, Proc. Amer. Math. Soc. 36 (1972), 187-190.

3. E. I. Grudo, Analytic theory of ordinary differential equations with deviating argument, Differencial'nye Uravnenija 5 (1969), 700-711. (Russian) MR 39 \#3118.

4. W. A. Harris, Jr., Holomorphic solutions of nonlinear differential equations at singular points, SIAM Studies in Appl. Math. 5 (1969), 184-187.

5. W. A. Harris, Jr., Y. Sibuya and L. Weinberg, Holomorphic solutions of linear differential systems at singular points, Arch. Rational Mech. Anal. 35 (1969), 245-248. MR 40 \#432.

6. F. Lettenmeyer, Über die an einer Unbestimmtheitsstelle regulären Lösungen eines Systemes homogener linearen Differentialgleichungen, S.-B. Bayer. Akad. Wiss. München Math.-Nat. Abt. (1926), 287-307.

7. D. I. Martynjuk, Integration by means of series of linear differential equations with deviating argument, Ukrain. Mat. Z. 18 (1966), 105-111. (Russian) MR 34 \#458.

Department of Mathematics, University of Missouri-Rolla, Rolla, Missouri 65401 